\title{
ALMA witnesses the assembly of first galaxies
}

\author{
Stefano Carniani \\ Scuola Normale Superiore, Piazza dei Cavalieri 7, I-56126 Pisa, Italy \\ email: stefano.carniani@sns.it
}

\begin{abstract}
Characterising primeval galaxies entails the challenging goal of observing galaxies with modest star formation rates ( $\mathrm{SFR}<100 \mathrm{M}_{\odot} \mathrm{yr}^{-1}$ ) and approaching the beginning of the reionisation epoch $(z>6)$. To date a large number of primeval galaxies have been identified thanks to deep near-infrared surveys. However, to further our understanding on the formation and evolution of such primeval objects, we must investigate their nature and physical properties through multi-band spectroscopic observations. Information on dust content, metallicity, interactions with the surrounding environment, and outflows can be obtained with ALMA observations of far-infrared (FIR) lines such as the [CII] at $158 \mu \mathrm{m}$ and [OIII] at $88 \mu \mathrm{m}$. Here, we, thus, discuss the recent results unveiled by ALMA observations and present new [CII] observations of BDF-3299, a star-forming galaxy at $z=7.1$ showing a spatial and spectral offset between the rest-frame UV and the FIR lines emission.
\end{abstract}

Keywords. galaxies: high-redshift, galaxies: ISM, infrared: ISM

\section{Introduction}

In the last decade deep optical and near-infrared imaging surveys carried out with the Hubble Space Telescope (HST) have identified a large number of galaxies within the Epoch of Reionization $(z>6)$. Such observations have shown that primeval galaxies are dramatically different from their low- $z$ counterparts being more compact, metal poor, and very clumpy likely due to intense merging activity (see review by Dayal \& Ferrara 2018). However, despite the remarkable progress, HST observations map only the restframe UV light powered by stellar population providing only partial information on galaxy assembly and evolution. It is thus fundamental to combine HST images with those obtained with the Atacama Large Millimetre/submillimetre array (ALMA) probing the interstellar medium (ISM), the main fuel of star formation activity in galaxies.

To date more than 40 star-forming galaxies with SFR $<100 \mathrm{M}_{\odot} \mathrm{yr}^{-1}$, which represent the typical galaxy population in the early Universe (e.g. Carniani et al. 2015; Robertson et al. 2015), have been studied with ALMA through the emission of the fine structure far-infrared (FIR) lines of [CII] at $158 \mu \mathrm{m}$ and [OIII] at $88 \mu \mathrm{m}$ (e.g., Maiolino et al. 2015; Pentericci et al. 2016; Carniani et al. 2017, 2018a,b; Matthee et al. 2018; Hashimoto et al. 2019). The [CII] line is emitted mainly from the neutral diffuse and partially ionised gas of the ISM, while the [OIII] arises from the ionised gas excited by star formation. ALMA observations have revealed that FIR lines are perfect tools to characterise ISM conditions, obtain precise redshift, and investigate gas kinematics. ALMA extended array configurations have also enabled to obtain images with angular resolution as high as $0.3^{\prime \prime}$ (Figure 1), which is comparable to the size of high- $z$ galaxies, and thus asses the galaxy morphology. For a large fraction of high- $z$ galaxies, [CII] emission breaks into multiple components associated to on-going merger processes (Carniani et al. 2018).

The high-resolution ALMA observations have also unveiled another puzzling scenario. For some galaxies the [CII] (or [OIII]) emission appears to be significantly offset both 


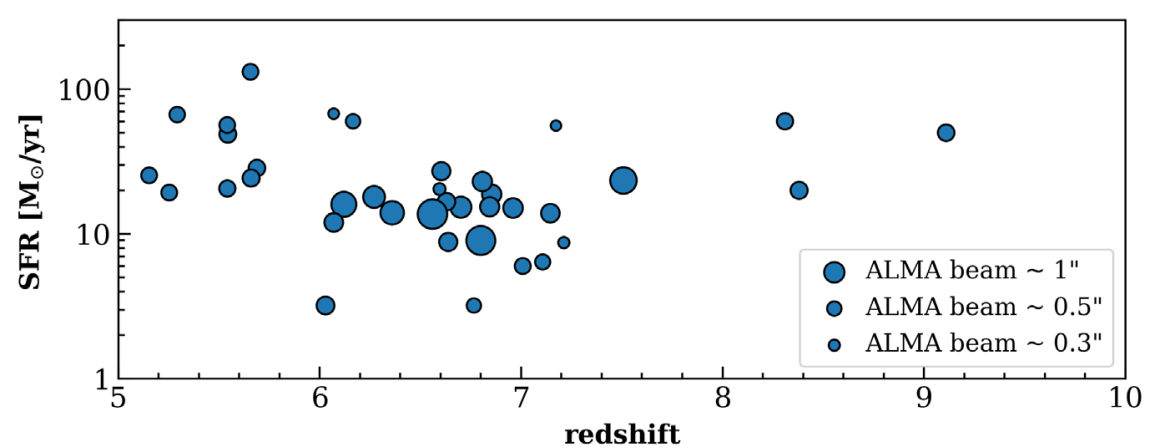

Figure 1. The redshift and SFR of high- $z$ galaxies observed with ALMA in either [CiI] or [Oin]. The size of the circle marks denotes the angular resolution (ALMA beam) of the observations.

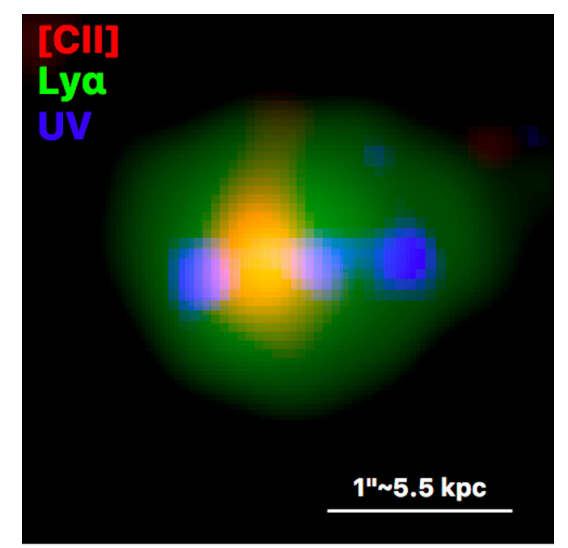

Figure 2. RGB image of Himiko, a star-forming galaxy at $z \sim 7$. Red, green, and blue images correspond to [CII], Ly $\alpha$, an d rest-frame UV.

in spatial position and in velocity relative to the Ly $\alpha$ and rest-frame UV emission. An example of displaced [CII] emission is illustrated in Figure 2 showing an RGB image of a Ly $\alpha$ emitter (LAE) a $z=6.6$, Himiko, comprised of three rest-frame UV bright clumps embedded in a extended Ly $\alpha$ nebula. The [CII] emission peaks in a region not detected in the rest-frame UV observations. According to current models, the [CII] deficit emission co-spatial to rest-frame UV region could be associated to intense stellar feedback removing or destroying molecular clouds and, thus regulating [CII] luminosity (Vallini et al. 2015; Ferrara et al. 2019). On the other hand, the displaced [Cir] line may trace either outflowing or accreting gas (Maiolino et al. 2015; Vallini et al. 2015; Pallottini et al. 2017). In the context of the origin of spatial and spectral offset of FIR lines, a key result has been provided by the ALMA observations of the star forming galaxy BDF-3299 at $z=7.1$ (Maiolino et al. 2015; Carniani et al. 2017) showing for the first time a clear evidence of displaced FIR emission. Here, we briefly review previous observations and new ALMA data of BDF-3299 confirming the detection of the spatially offset [CII] emission.

\section{BDF-3299}

BDF-3299 is a spectroscopically confirmed LAE at $z=7.109$ with $\mathrm{SFR} \approx 6 \mathrm{M}_{\odot} \mathrm{yr}^{-1}$ (Vanzella et al. 2011). The galaxy is located in the Bremer Deep Field (BDF) where two additional LAEs, within a projected distance of $\sim 2 \mathrm{Mpc}$ from BDF-3299, have been recently identified indicating that the $\mathrm{BDF}$ area is an overdense, reionized region in the 
Table 1. Properties of ALMA [CII] observations

\begin{tabular}{|c|c|c|c|}
\hline $\begin{array}{l}\text { Obs. } \\
\text { (a) }\end{array}$ & $\begin{array}{l}\sigma_{\text {cont }} \\
\text { (b) }\end{array}$ & $\begin{array}{c}\sigma_{\text {line }} \\
(\mathrm{c})\end{array}$ & $\begin{array}{l}\text { beam } \\
\text { (d) }\end{array}$ \\
\hline Cycle 1 & 8 & 62 & $0.8^{\prime \prime} \times 0.6^{\prime \prime}$ \\
\hline Cycle 2 & 11 & 90 & $0.3^{\prime \prime} \times 0.2^{\prime \prime}$ \\
\hline Cycle $1+2$ & 7 & 58 & $0.6^{\prime \prime} \times 0.5^{\prime \prime}$ \\
\hline Cycle 4 & 11 & 70 & $0.6^{\prime \prime} \times 0.5^{\prime \prime}$ \\
\hline
\end{tabular}

$z \sim 7$ Universe (Castellano et al. 2016, 2018). Among the three LAEs, BDF-3299 is the faintest in Ly $\alpha$ with a flux of $1.2 \times 10^{-17} \mathrm{erg} \mathrm{s}^{-1} \mathrm{~cm}^{-2}$ and $\mathrm{EW}(\operatorname{Ly} \alpha)=50 \AA$.

During ALMA Cycle 1 we led an observational program to investigate the strength of the [CII] line of BDF-3299 (Maiolino et al. 2015). The deep observations ( 8h on source) have revealed a carbon emission line consistent with the redshift of the galaxy. The [CII] line is slightly blueshifted by $64 \mathrm{~km} \mathrm{~s}^{-1}$ relative to Ly $\alpha$ redshift, which is expected since the blue side of Ly $\alpha$ is heavily absorbed by intervening intergalactic medium, hence artificially redshifted relative to the actual rest frame. However, the detection is offset by $0.7^{\prime \prime}(\sim 4 \mathrm{kpc})$ relative to the rest UV counterpart. This has been the first detection of displaced [CII] emission in the distant Universe and such an offset is not ascribed to astrometric uncertainties, as astrometry has been checked through serendipitous sources. These results seem to support model predictions expecting that molecular clouds in the central parts of primordial galaxies are rapidly disrupted by stellar feedback. As a result, [CII] emission mostly arises from more accreting/satellite clumps of neutral gas (Vallini et al. 2015).

In Carniani et al. (2017) we have reported new follow-up [CII] observations carried out with ALMA in Cycle 2 with the goal of obtaining a [CII] map at a resolution $\sim 3$ times higher than previous observations. Unfortunately, the observations were affected by various instrumental issues (differential timing among the different antennae, resulting in phase shifts across the field of view) and the sensitivity of the delivered data was worse than requested (Table 1). Given the low sensitivity and the high angular resolution of observations $\left(\sim 0.3^{\prime \prime}\right.$, Table 1$)$, most of the diffuse [CiI] emission is resolved out and only $\sim 20 \%$ of the total emission observed by Maiolino et al. (2015) is detected in the new dataset, indicating that the gas is extended on scales larger than $1 \mathrm{kpc}$. By combining the Cycle 1 and 2 datasets we have obtained a deeper [CII] image at a intermediate angular resolution with respect to the two separate datasets (Table 1). The combined image revealed that the displaced [CII] emission has a clumpy structure, likely associated to a interacting system and/or a result of a feedback process (Carniani et al. 2017).

In ALMA Cycle 2 we also proposed [OIII] observations of BDF-3299. [OIII] emission was detected at high significance $(\sim 9 \sigma)$ and spatially offset relative to the optical counterpart in the same direction as the [CII] clump, but not completely overlapping with the bulk of the [CII] emission (see Fig. 4 by Carniani et al. 2017). The central velocity of the [OIII] is redshifted by about $440 \mathrm{~km} \mathrm{~s}^{-1}$ with respect to the Ly $\alpha$ peak and about 500 $\mathrm{km} \mathrm{s}^{-1}$ relative to the [CII] emission, indicating that the two emission FIR lines, [OIII] and $[\mathrm{CII}]$ are tracing two different clumps next to BDF-3299. By comparing our data with models by Vallini et al. (2017) we have concluded that the spatial and spectral offset [OIII] line cannot be excited by the UV radiation coming from BDF-3299, but the oxygen line must be excited by in-situ star formation that is not observable in current rest-frame UV images due to dust extinction (Carniani et al. 2017). 

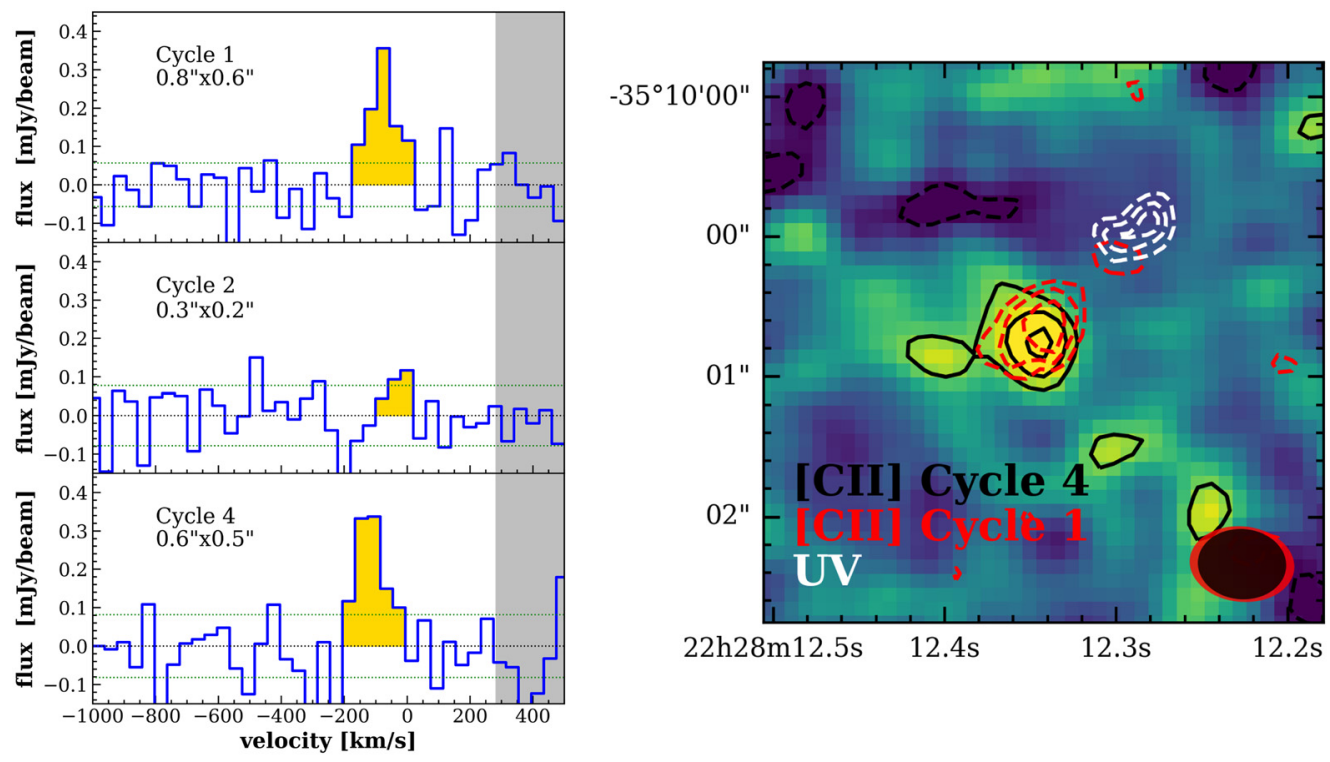

Figure 3. Left: [CII] spectra of BDF-3299 extracted from the three individual datasets The rms noise levels are shown by the black dotted lines. The grey shaded region indicates the part of the spectrum affected by higher noise because of atmospheric absorption. Right: [CII] map of BDF-3299 from new Cycle 4 observations (black contours are in steps of $1 \sigma$, starting at $2 \sigma$ ). Blue contours trace the [CII] surface brightness from the Cycle 1 data (Maiolino et al. 2015) and contours are at levels 2, 3 and 4 times noise per beam. The white contours trace the Y-band emission (UV-rest frame). The ALMA synthesised beam of Cycle 1 and 4 observations are shown in red and blue in the bottom-right corner, respectively.

The complex scenario revealed by ALMA in the $z=7.1$ galaxy is consistent with expectations by recent models and cosmological simulations (Vallini et al. 2015; Katz et al. 2017; Pallottini et al. 2017, 2019). The observational properties can be interpreted as a primeval system in the process of being assembled, where different emission lines trace distinct components, each possibly characterised by a different metallicity and/or excitation (ionisation parameter). In BDF-3299 the observable UV light is associated with the least obscured region, either because recently accreted, hence with low metallicity, or because strong feedback has removed the bulk of dust and gas content; both scenarios would account for the weakness of FIR line emission at the location of the optical image.

Cycle 1 and 2 ALMA observations are clearly tracing the process of assembly of a primeval galaxy and supporting the expectation of theoretical models. However, our capability of constraing galaxy formation models and properties of the intergalactic and circumgalactic medium is still limited by the sensitivity of our observations. In order to overcome this, we proposed deeper observations in [CII] and [OIII] of BDF-3200 in Cycle 4 aimed at achieving sensitivities twice higher than previous ones and studying the gas kinematics. Unfortunately [OIII] observations were not performed and the [CII] program was observed only for half of the requested time reaching a sensitivity comparable to the other previous datasets (see Table 1).

The displaced [CII] emission is detected in the new individual dataset with a level of significance $>4.5 \sigma$ and its location is in agreement with previous observations (Figure 3 ). The line width and the centroid are slightly different from those reported by Maiolino et al. (2015) and Carniani et al. (2017), but they are consistent within the uncertainties. We note that the angular resolution of Cycle 4 observations is 1.6 times better than in Cycle 1 and some of the diffuse emission could be resolved out in the new observations 
resulting in a slightly different line profile. At the location of the [OIII] clump any emission line has been detected with a level of significance higher than $2 \sigma$. A detailed analysis of the new data and the results obtained by combining all ALMA observations from the various cycles are discussed in a separate (Carniani et al. in prep.). The new observations of BDF-3299 confirm our previous results indicating the [CII] emission line traces the diffuse and accreting gas around high- $z$ galaxies. Intense feedback mechanisms may destroy molecular gas from the central galaxy and explain the deficit of [CII] observed in several primeval galaxies. We note that the detection of displaced [CII] emission is not limited to this galaxy, but this scenario is observable in several other $z>6$ star-forming galaxies. By taking into account that in some cases the [CII] line is not associated to the UV counterpart, we have recently found that the $\mathrm{L}_{[\mathrm{CII}]}-S F R$ relation at early epochs is fully consistent with the local relation (Carniani et al. 2018)

These preliminary results suggest that future deep ALMA observations in both [OIII] and [CII] applied to a large sample will be crucial to put stringent constrains on ISM properties and galaxy formation models.

\section{Summary and conclusions}

We have briefly summarised recent stat-of-art of ALMA observations targeting $z>6$ star-forming galaxies. The ALMA extended array configurations have enabled us to reach high-angular resolution and exploit FIR fine structure emission lines, such as [CII] and [OIII], as powerful diagnostics to assess the morphology of primeval galaxies. In particular ALMA observations have revealed that in several galaxies the [CII] emission is not associated with any UV counterpart at the current limits. In this context, we have reviewed ALMA observations of BDF-3299 which was the first high- $z$ star-forming galaxy showing a spatial offset between the FIR lines and UV emission. Finally we have discussed the preliminary results obtained by new [CII] observations of BDF-3299 confirming that the displaced [CII] emission is not due a glitch in the observation, but is a real detection, tracing circumgalactic gas in accretion or ejected by the main galaxy.

\section{Acknowledgements}

The results presented in this paper were obtained in collaboration with several other astronomers, and in particular: A. Ferrara, R. Maiolino, A. Pallottini, L. Vallini, S. Gallerani, and L. Pentericci. This work is supported by the ERC Advanced Grant INTERSTELLAR H2020/740120. This work reflects only the author's view and the European Research Commission is not responsible for information it contains.

\section{References}

Dayal \& Ferrara, 2018, PhR, 780, 1D

Carniani, S., Maiolino, R., De Zotti, G., et al. 2015, A\&\&A, 584A, 78C

Carniani, S., Maiolino, R., Pallottini, A., et al. 2017, A\&A A, 605A, 42C

Carniani, S., Maiolino, R., Smit, R., et al. 2018a, ApJ, 854L, 7C

Carniani, S., Maiolino, R., Amorin, R., et al. 2018b, MNRAS, 478, 1170C

Castellano, M., Dayal, P., Pentericci, L., et al. 2016, ApJ, 818L,3C

Castellano, M., Pentericci, L., Vanzella, E., et al. 2018, ApJ, 863L, 3C

Ferrara, A., Vallini, L., Pallottini, A., et al. 2019, MNRAS, 489, 1F

Hashimoto, Takuya, Inoue, Akio K., Mawatari, Ken., et al. 2019, PASJ, tmp, 70H

Kohandel, M., Pallottini, A., Ferrara, A., et al. 2019, MNRAS, 487, 3007K

Maiolino, R., Carniani, S., Fontana, A., et al. 2015, MNRAS, 452, 54M

Matthee, J., Sobral, D., Boone, F., et al. 2017, ApJ, 851, 145M

Pallottini, A., Ferrara, A., Gallerani, S., et al. 2017, MNRAS, 465, 2540P

Pallottini, A., Ferrara, A., Decataldo, D., et al. 2019, MNRAS, 487, 1689P 
Pentericci, L., Carniani, S., Castellano, M., et al. 2016, ApJ, 829L, 11P

Robertson, B. E., Ellis, R. S., Furlanetto, S. R., \& Dunlop, J. S. 2015, ApJ, 802, L1

Vallini, L., Gallerani, S., Ferrara, A., et al. 2015, ApJ, 813, 36V

Vallini, L., Ferrara, A., Pallottini, A., et al. 2017, MNRAS, 467, 1300V

Vanzella, E., Pentericci, L., Fontana, A., et al. 2011, ApJ, 730L, 35V 